\title{
Large community-acquired Legionnaires' disease outbreak caused by Legionella pneumophila serogroup 1, Italy, July to August 2018
}

\author{
Marino Faccini ${ }^{1,2}$, Antonio Giampiero Russo ${ }^{1,2}$, Maira Bonini ${ }^{1,2}$, Sara Tunesi ${ }^{1}$, Rossella Murtas ${ }^{1}$, Monica Sandrini ${ }^{1}$, Sabrina \\ Senatore ${ }^{1}$, Anna Lamberti ${ }^{1}$, Giorgio Ciconali ${ }^{1}$, Serafina Cammarata ${ }^{1}$, Eros Barrese ${ }^{1}$, Valentina Ceriotti ${ }^{1}$, Sonia Vitaliti ${ }^{1}$, Marina \\ Foti $^{1}$, Gabriella Gentili ${ }^{1}$, Elisabetta Graziano ${ }^{1}$, Emerico Panciroli ${ }^{1}$, Marco Bosio ${ }^{1}$, Maria Gramegna ${ }^{3}$, Danilo Cereda ${ }^{3}$, Carlo \\ Federico Perno ${ }^{4}$, Ester Mazzola ${ }^{4}$, Daniela Campisi ${ }^{4}$, Gianuario Aulicino5 , Silvana Castaldi ${ }^{5,6}$, Antonietta Girolamo ${ }^{7}$, Maria \\ Grazia Caporali7 , Maria Scaturro ${ }^{7}$, Maria Cristina Rota ${ }^{7}$, Maria Luisa Ricci ${ }^{7}$ \\ 1. Agency for Health Protection of Metropolitan Area of Milan (ATS), Milan, Italy \\ 2. These authors contributed equally to this article and share first authorship \\ 3. Direzione Generale Welfare, Unità Organizzativa Prevenzione, Lombardy Region, Milan, Italy \\ 4. Department of Laboratory Medicine, Hospital Niguarda, Milan, Italy \\ 5. Department of Biomedical Sciences for Health, Post Graduate School in Public Health, University of Milan, Milan, Italy \\ 6. Fondazione IRCCS Ca' Granda Ospedale Maggiore Policlinico, Milan, Italy \\ 7. Department of Infectious Diseases, Istituto Superiore di Sanità, Rome, Italy
}

Correspondence: Marino Faccini (mfaccini@ats-milano.it)

Citation style for this article:

Faccini Marino, Russo Antonio Giampiero, Bonini Maira, Tunesi Sara, Murtas Rossella, Sandrini Monica, Senatore Sabrina, Lamberti Anna, Ciconali Giorgio , Cammarata Serafina, Barrese Eros, Ceriotti Valentina, Vitaliti Sonia, Foti Marina, Gentili Gabriella, Graziano Elisabetta, Panciroli Emerico, Bosio Marco, Gramegna Maria, Cereda Danilo, Perno Carlo Federico, Mazzola Ester, Campisi Daniela, Aulicino Gianuario, Castaldi Silvana, Girolamo Antonietta, Caporali Maria Grazia , Scaturro Maria , Rota Maria Cristina, Ricci Maria Luisa . Large community-acquired Legionnaires' disease outbreak caused by Legionella pneumophila serogroup 1, Italy, July to August 2018. Euro Surveill. 2020;25(20):pii=1900523. https://doi.org/10.2807/1560-7917.ES.2020.25.20.1900523

Article submitted on 14 Aug 2019 / accepted on 19 Dec 2019 / published on 21 May 2020

In July 2018, a large outbreak of Legionnaires' disease (LD) caused by Legionella pneumophila serogroup 1 (Lp1) occurred in Bresso, Italy. Fifty-two cases were diagnosed, including five deaths. We performed an epidemiological investigation and prepared a map of the places cases visited during the incubation period. All sites identified as potential sources were investigated and sampled. Association between heavy rainfall and LD cases was evaluated in a case-crossover study. We also performed a case-control study and an aerosol dispersion investigation model. Lp1 was isolated from 22 of 598 analysed water samples; four clinical isolates were typed using monoclonal antibodies and sequence-based typing. Four Lp1 human strains were ST23, of which two were Philadelphia and two were France-Allentown subgroup. Lp1 ST23 France-Allentown was isolated only from a public fountain. In the case-crossover study, extreme precipitation 5-6 days before symptom onset was associated with increased LD risk. The aerosol dispersion model showed that the fountain matched the case distribution best. The case-control study demonstrated a significant eightfold increase in risk for cases residing near the public fountain. The three studies and the matching of clinical and environmental Lp1 strains identified the fountain as the source responsible for the epidemic.

\section{Background}

Legionella pneumophila (Lp) is a Gram-negative bacterium responsible for a severe pneumonia named
Legionnaires' disease (LD). This infection represents $1.9 \%$ of all community-acquired pneumonia cases, $4.0 \%$ of hospitalised cases and $7.9 \%$ of cases requiring admission to intensive care units [1]. The case fatality rate of LD ranges from $5 \%$ to $30 \%$ during outbreaks but can reach up to $50 \%$ in nosocomial cases or if antibiotic treatment is delayed [2]. The European Legionnaires' disease Surveillance Network (ELDSNet) has reported an increase in age-standardised LD notification rates in the period 2011to 2017 [3]. The same trend has been observed in Italy, with incidence rates increasing from 1.56 per 100,000 in 2011 to 4.9 per 100,000 in 2018 [4]. LD occurs predominantly in the elderly with chronic lung disease; immunosuppression and smoking as the most important risk factors. The incubation period ranges between 2 and 10 days from the, often nonspecific, initial symptoms. Infection occurs through inhalation of aerosols produced by contaminated water systems [3]. Outbreaks have been linked to a variety of aerosol-producing devices, such as cooling towers, evaporative condensers and spa pools $[5,6]$.

Improvements in diagnosis and surveillance may partly explain the increase in reported LD cases worldwide, however, several studies have suggested that higher atmospheric temperatures and changes in rainfall patterns may play a significant role [7-9]. Moreover, age-standardised rates are increasing, with a greater number of fragile individuals who are at higher risk of acquiring the infection [4]. 
Confirmed cases of Legionnaires' disease by date of symptom onset, Bresso, Italy, 10-31 July 2018 (n = 52)

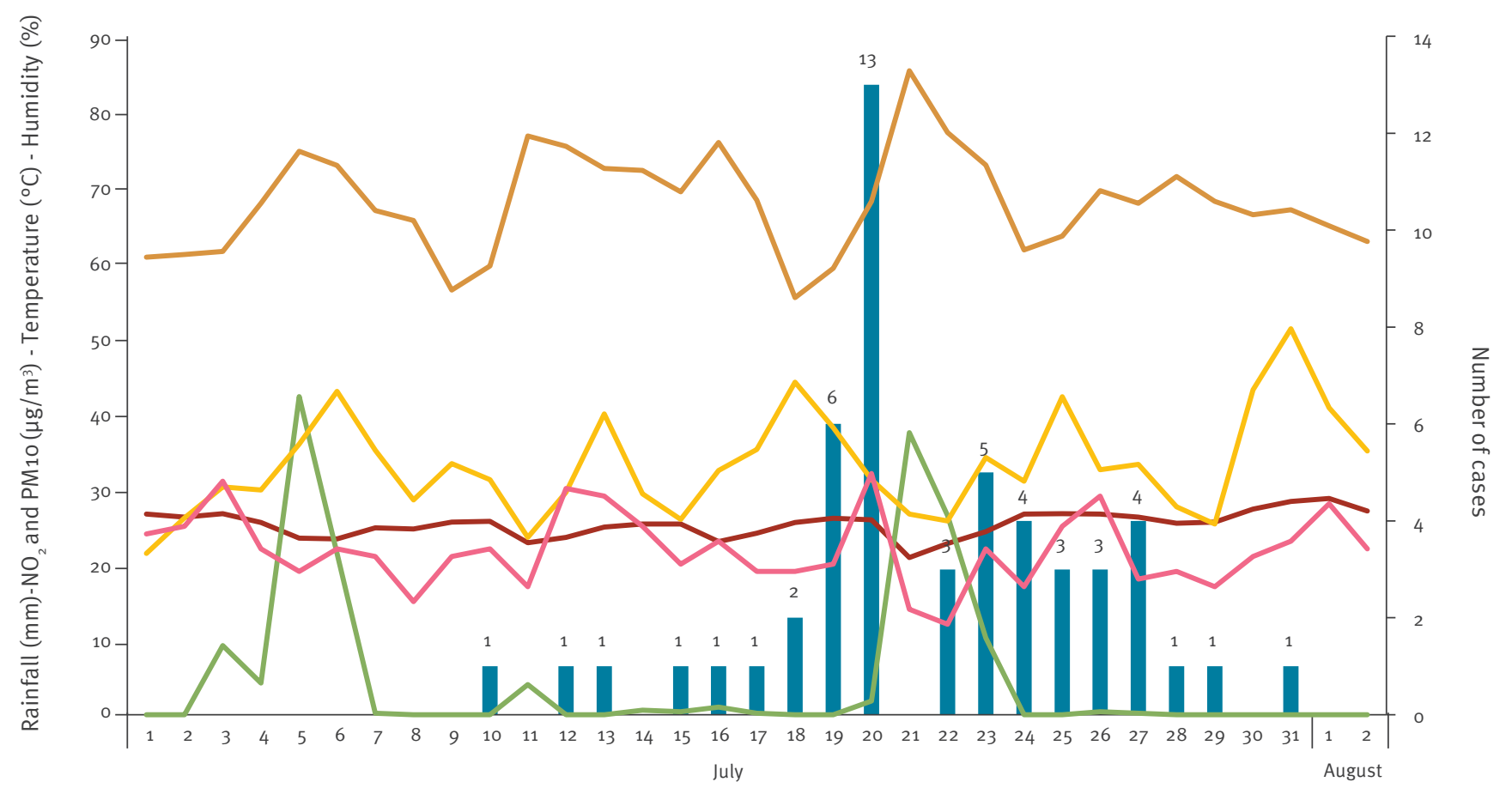

Date in 2018

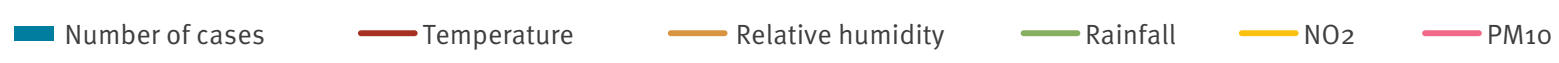

Temperature, relative humidity, rain measure and pollution ( $\mathrm{PM} 10$ and $\mathrm{NO}_{2}$ ) are also shown.

Bresso is a town (3.38 $\mathrm{km}^{2}$ with 26,285 inhabitants) located near Milan in Lombardy, the region with the third highest LD incidence in Italy (10 cases per 100,000 people in 2018) [10]. In 2014, an LD cluster occurred in Bresso involving six cases within a period of 20 days. All cases were men aged $58-78$ years, one of whom died. The only clinical isolate available was typed as ST23. The source of infection was not identified.

In July 2018, a new and larger outbreak of LD occurred in Bresso, involving 52 cases. The aim of this paper was to report epidemiological, microbiological and environmental investigations and describe factors that contributed to the outbreak.

\section{Outbreak detection}

In Bresso, the number of LD cases reported in the period 2015 to 2017 was between one and three cases per year. Thus, when the Agency for Health Protection of the Metropolitan Area of Milan (ATS) received the notification of three LD cases occurring in citizens living in Bresso between 16 and 17 July 2018, the suspicion of an epidemic cluster arose immediately. Additional cases were soon notified and the suspicion was confirmed. A multidisciplinary team including epidemiologists, public health operators, medical doctors and microbiologists was established to control the outbreak and to conduct epidemiological and environmental investigations.

\section{Methods}

\section{Case definition and epidemiological investigation}

A probable outbreak-associated case was defined as a person with confirmed or probable LD according to the European Union (EU) case definition [11] with symptom onset between 10 and 31 July 2018, who lived in, or visited, the outbreak area (the town of Bresso) in the 10 days before symptom onset. Confirmed nosocomial cases or cases who had travelled outside Bresso for the entire incubation period were not considered.

An epidemiological investigation was performed and information regarding demographic, clinical and risk factors was collected. A map of the routes and places visited in the city of Bresso during the incubation period was drawn for each case. The information collected was used for geolocalisation studies and routes and places were plotted using ArcGIS software (esri, Redlands, United States (US)). 
Mapping of Legionnaires' disease cases: paths collected through individual questionnaires and aerosol dispersion model with potential sources' plumes, Bresso, Italy, 10-31 July $2018(\mathrm{n}=52)$
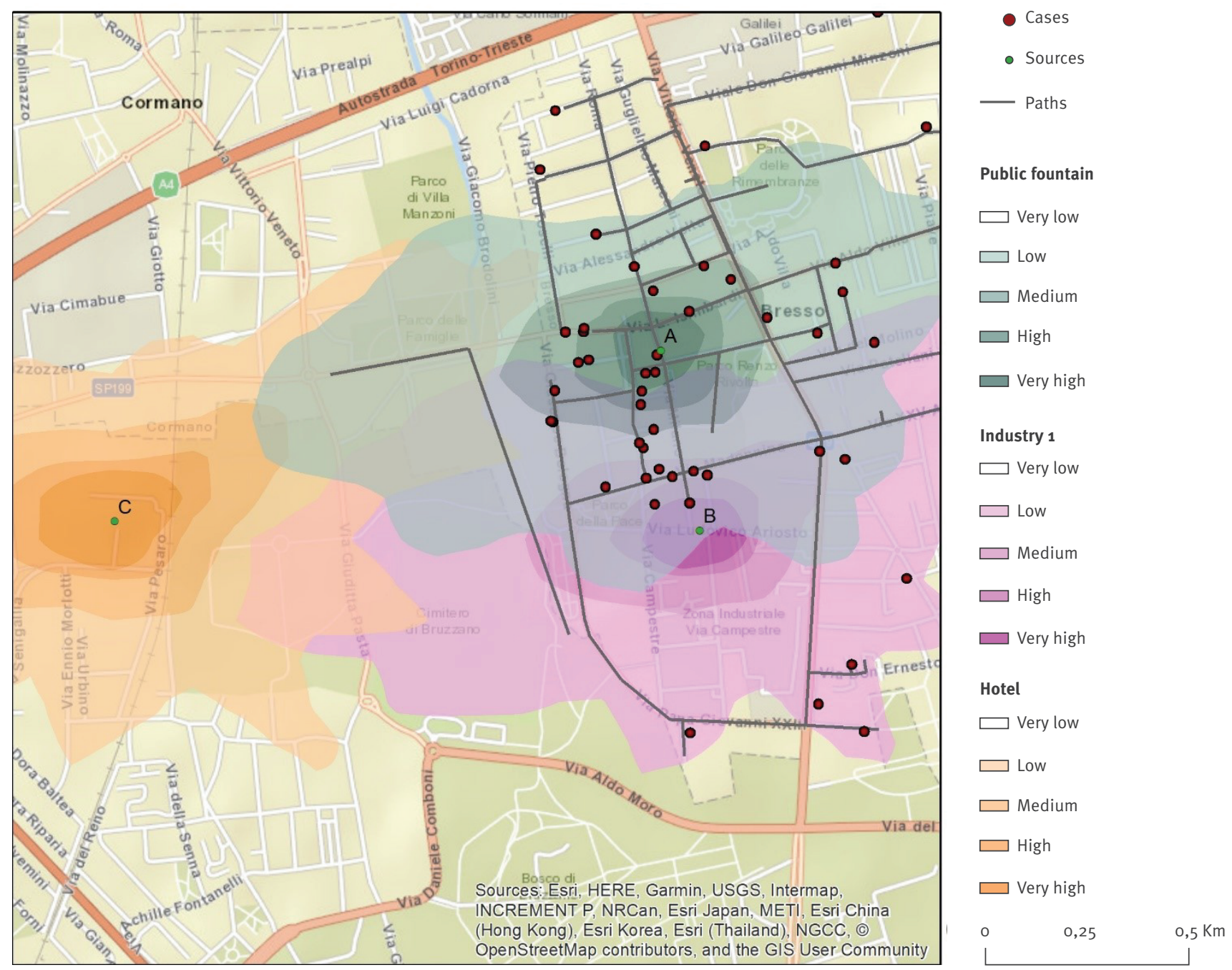

Public fountain (site A), cooling tower of Industry 1 (site B) and hotel (site C). Different colours represent the intensity of concentration within each plume.

\section{Environmental investigation}

All sites identified as potential sources of the disease were inspected and sampled. The sites were chosen based on: the place of residence and main locations frequented by the cases (e.g. malls, recreational facilities and squares) and highly crowded outdoor areas with aerosol-producing devices (e.g. cooling systems, sprinklers and fountains). The municipal water main line was also sampled at selected points. All potential sources were entered into the geographical information system (GIS).

\section{Microbiological analysis of clinical and environmental samples}

LD cases were diagnosed by both urinary antigen test (Binax Now; Alere-Abbott, Scarborough, US) and culture of respiratory secretions or pulmonary tissues. For culture examination, we used agar plates with buffered charcoal yeast extract (BCYE-a; Thermo Fisher Diagnostics Limited - Altrincham, United Kingdom) and
BCYE with selective antibiotic (MWY; Thermo Fisher Diagnostics).

Water samples were tested by both real-time PCR and culture. Real-time PCR assays were performed using a validated commercial kit (iQ-Check Screen Legionella spp and iQ-Check Screen L. pneumophila; Biorad, Marnes-la-Coquette, France), according to ISO/ TS 12869, 2012 [12]. Water samples positive for Lp by qualitative real-time PCR (automatic cycle threshold $(\mathrm{CT})$, positive $\leq 43 \mathrm{CT}$ ) were also analysed by culture in order to isolate Legionella strains. For culture, $1 \mathrm{~L}$ of water was analysed according to ISO 11731:2017 using a detection limit of 100 colony-forming units (CFU)/L) [13]. In some cases, to increase Legionella recovery, biofilms or sediments were sampled and analysed according to ISO 11731:2017.

Colonies from clinical and environmental samples were identified by latex agglutination test (Thermo Fisher 
TABLE

Legionella pneumophila serogroup 1 colonies isolated from different environmental sites, Bresso, Italy, 10-31 July 2018 ( $\mathrm{n}=8$ isolates $)$

\begin{tabular}{|l|l|l|l|}
\hline Site & Sampling point & MAb subgroup & ST \\
\hline $\begin{array}{l}\text { Public fountain } \\
\text { (Site A) }\end{array}$ & Collection basin & $\begin{array}{l}\text { France/ } \\
\text { Allentown }\end{array}$ & 23 \\
\hline House 1 & Shower & Oxford & 1 \\
\hline House 2 & Shower & Benidorm & 2,695 \\
\hline Hotel (Site C) & Cooling tower pond & Philadelphia & 1 \\
\hline Industry 1 (Site B) & Cooling tower pond & Bellingham & 37 \\
\hline Industry 1 (site B) & Cooling tower pond & Bellingham & 1 \\
\hline Industry 1 (site B) & Cooling tower pond & Olda & 1 \\
\hline Industry 2 & Tap of toilet & Philadelphia & 1 \\
\hline
\end{tabular}

MAb: monoclonal antibody; ST: sequence type.

Diagnostics Limited - Altrincham). All Lp serogroup 1 (Lp1) colonies identified were typed using monoclonal antibodies (MAb) of the Dresden Panel [14] and genotyped by sequence-based typing (SBT) [15].

\section{Case-crossover design}

To evaluate the associations between heavy rainfall and LD cases, a unidirectional case-crossover design $[16,17]$ was adopted. For each subject, the case-day was identified as the day of LD symptom onset. Controldays were defined as the period ranging from 10 to 3 days before the case-day. For example, for a subject with symptoms starting on 15 July, the case-day was identified as 15 July and the control days ranged from 5 to 11 July 2018.

Each case-day was matched with its control-days so that adjustment for usual confounding, such as age and sex, was assured by design. The occurrence of extreme precipitation events (defined as days with a precipitation higher than $31 \mathrm{~mL} / \mathrm{h}$ ) was compared between case-days and control-days. Thirty $\mathrm{mL}$ of daily cumulative rainfall was used as a cut-off point to identify heavy rainfall, and a dummy variable was used as exposure. Climatological and air quality components were included as covariates.

Hourly environmental data concerning precipitation, air temperature and relative humidity, measured between July and August 2018, were obtained from a monitoring station of the Regional Environmental Protection Agency (ARPA), located $2 \mathrm{~km}$ from Bresso. Mean daily data on particulate matter $10 \mu \mathrm{m}$ or less in diameter (PM10) and on nitrogen dioxide $\left(\mathrm{NO}_{2}\right)$ were obtained from a monitoring station located in Bresso [11].

\section{Case-control study}

A retrospective case-control study was also performed. Cases were defined as reported in the case definition and up to four controls were selected for each case $[18,19]$. Controls were randomly selected from the local residency register and matched with cases for sex, age and history of chronic pathology (renal failure, diabetes, etc). Each control was matched with a case for all the conditions included as matching variables, and residence was geolocalised using the ArcGIS software. To investigate the potential source of infection, we analysed place of residence and main meeting points such as malls, fountains and shops. Exposure was defined as the distance of each case and its controls from each potential source positive for Lp1, hypothesising that the prevalence of cases living close to the source was different from the prevalence of controls. Distance from each source was divided in tertiles and the third tertile was used as the reference group.

\section{Aerosol dispersion investigation}

Dispersion models were used to estimate the exposed population following a potential release and to infer potential source sites from the pattern of observed infections. We investigated the transport and dispersion of aerosol during the infection period (5-20 July 2018) using the LAPMOD modelling system [20]. This is a three-dimensional, non-stationary, Lagrangian particle model used to simulate the atmospheric dispersion of inert or radioactive gases and aerosols, over complex terrain, emitted from the potential source of infection.

To improve the resolution of terrain topography and land use, the meteorological input of the LAPMOD model was the output field generated by the diagnostic meteorological model CALMET [21] from 1 to 31 July. The geophysical variables necessary for the LAPMOD model estimation of deposition flows (i.e. roughness or soil occupation category) also come from CALMET.

CALMET was generated using wind temperature, direction and speed values at various altitudes of the troposphere, provided by the radiosonde at Milan Linate airport, the prognostic meteorological field produced by the weather research and forecasting model and the surface temperature from Bresso ARPA monitoring station.

The weather field used was validated by reconstructing the wind rose at the nearest ARPA control unit, not included in the calibration phase.

Inhalation of Legionella-contaminated droplets occurs when the aerosol size is smaller than $10 \mu \mathrm{m}[5,22]$. For this reason, we investigated the dispersion of PM10 from a plausible pipe diameter. Emission height, output velocity and temperature were introduced in the model according to source characteristics. We simulated two different types of dispersion: cooling towers and fountains. For cooling towers, we hypothesised a pipe diameter of $1 \mathrm{~m}$ positioned on the roof of the corresponding building [23]. Output velocity was $3 \mathrm{~m} / \mathrm{s}$, emission rate was $100 \mathrm{~g} / \mathrm{s}$ and, being hot emissions, a temperature $10^{\circ} \mathrm{C}$ higher than the temperature recorded by the nearest ARPA control units, assessed every hour. For fountains, we simulated a cold emission of $100 \mathrm{~g} / \mathrm{s}$ 
positioned at ground level and a diameter of $1 \mathrm{~m}$. Since the real emission rates were not available, we hypothesised an emission rate of $100 \mathrm{~g} / \mathrm{s}$ for each potential source [23]. Dry and wet depositions were simulated in both models. We estimated the mean concentration of emissions and defined levels of intensity from very low to very high. Plumes were visualised using ArcGIS.

\section{Statistical analysis}

Odds ratios were calculated using conditional logistic regression with LD diagnosis as outcome, distance from potential source in case-control and extreme precipitation in case-crossover studies.

Climatological and air quality components such as $\mathrm{NO}_{2}$, PM10, temperature and relative humidity, were considered as potential confounders.

All statistical analyses were performed using SAS version 9.4 .

\section{Ethical statement}

Ethical approval was not necessary because the data used for the study were collected as part of the infectious disease surveillance programmes defined by national legislation.

\section{Results}

\section{Epidemiological investigation}

Fifty-two confirmed LD cases among residents in Bresso were notified between 10 and 31 July, with a peak on 20 July (Figure 1).

The mean patient age was 73 years (range: 33-95; median: 75$)$ and 32 (61.5\%) were men. Forty-six cases presented at least one individual risk factor for LD; 41 patients had an underlying disease and 22 patients had two or more chronic conditions. Eighteen cases reported cigarette smoking, which was the only risk factor in six cases. Forty cases were hospitalised and five died. The case fatality rate was $9.6 \%$.

Most of the cases reported to have visited the central area of the town during the incubation period, which also coincided with the place of residence of many cases $(n=41)$.

\section{Microbiological investigation}

All 52 LD cases were confirmed by urinary antigen test and Lp1 was isolated by culture of respiratory secretions in four cases. The SBT and MAb typing was carried out both for clinical and environmental strains. Of the four Lp1 ST23 human strains two were Philadelphia and two were France-Allentown subgroup. Typing data of Lp environmental isolates are reported in the Table.

Overall, 101 sites ( 52 patients' homes and 49 potential sources) were sampled and 598 water samples were collected.
Five of 52 patients' houses were found to be contaminated by Lp and only two houses resulted culture-positive for LP1 $(3,100$ and 26,000 CFU/L). Real-time PCR was positive in four residences, including the two positive by culture.

Seven of 49 sites identified as potential sources were found positive for Lp by culture, while real-time PCR gave positive results in 10 sites including the seven positive by culture. Two of 11 inspected cooling towers resulted positive for Lp1 with 1,000 CFU/L and 6,500 CFU/L. Moreover, a decorative fountain located in a public garden of the town, where many inhabitants stopped or passed by, was sampled on 25 July 2018 and was found positive for Lp1 (1,000-2,000 CFU/L). Sampling of the fountain was repeated 1 month and 3 months later and was found contaminated with Lp1 at 2,000 CFU/L and 50,000 CFU/L, respectively. Three additional decorative fountains located in the outbreak area were sampled: two were negative ( $100 \mathrm{CFU} / \mathrm{L}$ ) and one positive for Lp5. The municipal water system was sampled at 10 different points, identified as critical, and these samples were all negative ( $100 \mathrm{CFU} / \mathrm{L})$.

\section{Case-crossover design}

A total of 52 LD case-days and 364 control-days were included in the analysis. Precipitation values ranged from 0.0 to $42.2 \mathrm{~mL}$ per day, with an average value of $3.7 \mathrm{~mL}$ per day over the study period and a cumulative value of $82.6 \mathrm{~mL}$. Two high precipitation events (> 31 $\mathrm{mL} / \mathrm{h}$ [24]) occurred during the outbreak period: 5 to 21 July 2018. Daily temperatures ranged between $20.9^{\circ} \mathrm{C}$ and $26.6^{\circ} \mathrm{C}$ with an average of $24.5^{\circ} \mathrm{C}$. The average relative humidity was $69.8 \%$ (range: $56.3-85.4$ ). The daily PM10 ranged from $12 \mu \mathrm{g} / \mathrm{m}^{3}$ to $32 \mu \mathrm{g} / \mathrm{m}^{3}$, with an average of $21 \mu \mathrm{g} / \mathrm{m}^{3}$. The daily $\mathrm{NO}_{2}$ ranged from 25.7 $\mu \mathrm{g} / \mathrm{m}^{3}$ to $39.9 \mu \mathrm{g} / \mathrm{m}^{3}$, with an average of $32.3 \mu \mathrm{g} / \mathrm{m}^{3}$. Adjusted analysis revealed that extreme precipitations, occurring 5 and 6 days before symptom onset, were associated with a fourfold increase in LD risk (OR $=4.03 ; 95 \%$ confidence interval $(\mathrm{Cl})$ : 0.76-21.3 and $\mathrm{OR}=3.58 ; 95 \% \mathrm{Cl}: 0.80-16.1$, respectively).

\section{Case-control study}

For each of the 48 cases, four matched controls were identified, i.e. 48 cases and 192 controls were included in the analysis. Of 101 sites analysed, Lp1 was only isolated from four non-residential sources: The mean distance of the public fountain from the place of residence was $391 \mathrm{~m}$ for cases and $646 \mathrm{~m}$ for controls (OR for first vs third tertile: $8.69 ; 95 \% \mathrm{Cl}: 3.04-24.83)$; the mean distance to Industry 1 was $777 \mathrm{~m}$ for cases and $661 \mathrm{~m}$ for controls (OR for first vs third tertile: $2.95 ; 95 \% \mathrm{Cl}$ : 1.15-7.59); the mean distance to the hotel was $1,859 \mathrm{~m}$ for cases and 2,153 $\mathrm{m}$ for controls (OR for first vs third tertile: 11.43 ; $95 \% \mathrm{Cl}: 3.46-37.69)$; and the mean distance to Industry 2 was $688 \mathrm{~m}$ for cases and $540 \mathrm{~m}$ for controls (OR for first vs third tertile: $0.90 ; 95 \% \mathrm{Cl}$ : $0.32-2.58)$. 


\section{Aerosol dispersion investigation}

Given that Industry 2 was not associated with LD in our case-control study, we limited the aerosol dispersion investigation to the public fountain (Site A), Industry 1 (Site B) and the hotel (Site C). A different map of aerosol dispersion over the area of interest was generated for each site contaminated with Lp1. The modelled dispersions were used to establish the proportion of cases exposed to the various potential sources by either living close to or visiting a location during the incubation period. The plume modelled from the public fountain (Site A) showed the best fit with the distribution of the cases (Figure 2).

\section{Outbreak control measures}

At the beginning of the investigation, emergency control measures were requested for all sites resulting positive by real-time PCR. In particular, the public fountain was immediately deactivated on 19 July, while cleaning and disinfection of the cooling towers were requested on 9 August. Once culture results were also available, disinfection was only requested for culture-positive sites. Owners of the sampled residences and sites at risk were informed of the sampling results and were asked to follow the best practices for Legionella control. No more cases occurred demonstrating the efficacy of control measures applied.

\section{Discussion}

The outbreak described here was the largest caused by Lp1 in Italy to date. A thorough environmental investigation excluded the municipal water system as source of the outbreak, since Legionella was undetectable in all samples collected. Nevertheless, the percentage of positive samples collected from cases' places of residence was small and no clinical strains were available for patients whose houses' samples resulted positive. Thus, matching from clinical and environmental isolates could not be performed.

The most common reported sources of Legionella outbreaks are cooling towers [25,26]. In Italy, two LD outbreaks caused by cooling towers occurred in 1995 and 2003, and another occurred in the period 2005 to 2008 , for which hidden cooling towers were suspected $[27,28]$. In the outbreak in Bresso, the SBT analysis showed that the human strains matched only with the environmental isolates from a small public fountain that had the same sequence type (ST23).

LD outbreaks have seldom been associated with decorative fountains $[29,30]$; in 2007, O'Loughlin et al. showed that a small fountain, without obvious aerosolgenerating capability, was identified as the source of 18 LD cases, and that removal of the fountain halted disease transmission. In addition to sociodemographic factors and associated chronic conditions, many factors influence LD transmission rates: bacterial concentration, distance from the source, high humidity, low atmospheric pressure and extreme rainfall events $[7,8,23,31]$.
The Bresso fountain was characterised by water recirculation, with little or no apparent aerosol production. Therefore, it was difficult to explain how the cases could have been infected. To identify the source of the outbreak, we carried out three studies.

The case-crossover study suggested that the heavy rainfalls which occurred 5 to 6 days before the onset of disease were associated with a fourfold increase in LD risk, even if not statistically significant. This time lag was slightly shorter than those reported in previous studies: (1 week [31] and 6-10 days [32]) and may have been related to particular atmospheric conditions in this area, such as the combination of temperature and high humidity that may promote Legionella spread.

The case-control study, performed to evaluate the association between the cases' places of residence and the distance from identified contaminated sites, showed a significant eightfold increase in risk for cases living near the public fountain. The OR for the hotel was 11.43; however, the strains isolated from the hotel cooling tower had a different ST than the clinical isolates. In addition, the hotel was located further the cases' places of residence than the fountain, and no cases were detected among hotel staff or guests.

Finally, an analytical study of the aerosol dispersion model applied to the potential sources showed that the fountain was the most probable source of infection.

A literature review has shown that the distance of Legionella transmission in an outbreak situation can vary from $500 \mathrm{~m}$ to $12 \mathrm{~km}$ [33]. In the Bresso outbreak, the potential source was identified at an average distance of $600 \mathrm{~m}$ from the cases.

A Lagrangian approach was used to model the discrete phase transport of bio-aerosol deposition. We hypothesised that the interaction between the high bacterial load in the recirculating water system that fed the fountain basin and the extreme rainfall of 5 and 21 July generated a bio-aerosol of contaminated particles which dispersed from the fountain, as simulated by the model.

We therefore consider it plausible that Legionella bioaerosols emitted from the fountain could have entered the surrounding buildings. This conclusion is reinforced by the fact that outdoor bio-aerosols have high penetration efficiency [5] and more than half the outbreak cases were sedentary individuals older than 75 years who reported spending most of their time at home or in indoor gathering places. The potential dispersion area of the public fountain was densely inhabited by cases.

All the studies conducted pointed to the public fountain as a potential source of the outbreak. In fact, the cleaning, disinfection and deactivation of the fountain on 19 July, halted the outbreak. 
It is difficult to explain why, after several years of operation, the fountain suddenly caused a large outbreak, but several elements must be considered. Firstly, the fountain is located in a public garden with connections to several unused water pipes. Secondly, the summer of 2018 was characterised by very high temperatures (hourly maximum temperature: $35^{\circ} \mathrm{C}$ ) and humidity (hourly maximum humidity: $86 \%$ ) throughout the months of June and July [34] and by two extreme rainfall events. This combination could have created fertile ground for Legionella growth and persistence.

As reported in a recent review, warm weather, rain and higher relative humidity may have an impact on the survival of airborne Legionella, as it has been shown to survive better at a relative humidity of $65 \%$ and less so at $30 \%$ or $90 \%$ relative humidity. Our meteorological data showed that relative humidity ranged from 60 to $80 \%$ throughout the outbreak period, increasing the risk of acquiring LD [35].

In conclusion, although at the beginning of the investigation, the public fountain did not appear as a plausible source of infection, the findings of all three studies, combined with the matching of the fountain isolates with the clinical isolates, identified the fountain as responsible for the outbreak.

\section{Study limitations}

The main limitation of the study is that only few clinical isolates were available, so that we could not ascertain if sources contaminated with other ST also contributed to the outbreak.

Another limitation was the information input to calculate the aerosol dispersion map. Changes in exit velocity and model characteristics resulted in large variations in emission concentrations and dispersion area. Furthermore, we investigated only the cases' places of residence and not all places visited, therefore underestimating exposure for some cases. A critical aspect was also the lack of robust methodology to ensure consistent bio-aerosol dispersion models.

Lastly, in the case-crossover study, although OR were not statistically significant, they were very close to 1 and the upper $\mathrm{Cl}$ limits were very high. Thus, increasing the sample size would probably have yielded significant results.

\section{Acknowledgements}

Health Protection Agency Metropolitan Area of Milan Legionnaires' Disease Investigation Teams: Public health unit; Infectious diseases surveillance unit; Laboratory of prevention; Occupational health and prevention unit; Epidemiological unit.

\section{Conflict of interest}

None declared.
Authors' contributions

Marino Faccini, Maria Luisa Ricci, Maria Cristina Rota: Coordinated and helped to draft the manuscript, made substantial contributions to acquisition of data.

Anna Lamberti and Sabrina Senatore: coordinated epidemiological investigations and helped to draft the manuscript.

Sonia Vitaliti: coordinated laboratory activities and relationships with other actors involved in environmental investigations.

Maria Grazia Caporali: helped to collect and elaborate epidemiological data.

Antonio Giampiero Russo: designed the study, perform casecrossover analysis and case-control study and took the lead in writing the first version of the paper.

Sara Tunesi and Rossella Murtas: planned and carried out the dispersion models and contributed to the writing of the manuscript.

Monica Sandrini: georeferenced cases/controls producing maps.

Danilo Cereda and Maria Gramegna: contributed to the implementation of the research.

Carlo Federico Perno: coordinated clinical laboratory activities.

Ester Mazzola and Daniela Campisi: followed and carried out analysis and interpretation of clinical laboratory results.

Marco Bosio, Emerico Panciroli, Giorgio Ciconali: contributed to the conception of the work.

Silvana Castaldi: discussed the results and contributed to the final manuscript.

Gianuario Aulicino: made substantial contribution to writing the manuscript with input from all authors.

Marina Foti: coordinated laboratory activities and relationships with other actors involved in environmental investigations; carried out laboratory activities and reports.

Gabriella Gentili and Elisabetta Graziano: followed and carried out laboratory activities and reports.

Maria Scaturro and Antonietta Girolamo: performed typing of isolated strains and reports.

Maira Bonini: coordinated the environmental investigation and the outbreak control measures.

Serafina Cammarata: helped the coordination of the environmental investigation and the outbreak control measures

Eros Barrese: supported the environmental investigations.

Valentina Ceriotti: helped the coordination of the environmental investigation and the outbreak control measures.

All authors read, reviewed and approved the final manuscript. 


\section{References}

1. Haubitz S, Hitz F, Graedel L, Batschwaroff M, Wiemken TL, Peyrani P, et al. Ruling out Legionella in community-acquired pneumonia. Am J Med. 2014;127(10):1010.e11-9. https://doi. org/10.1016/j.amjmed.2014.03.042 PMID: 24813862

2. Garrison LE, Kunz JM, Cooley LA, Moore MR, Lucas C, Schrag S, et al. Vital signs: deficiencies in environmental control identified in outbreaks of Legionnaires' disease - North America, 2000-2014. MMWR Morb Mortal Wkly Rep. 2016;65(22):576-84. https://doi.org/10.15585/mmwr. mm6522e1 PMID: 27281485

3. European Centre for Disease Prevention and Control (ECDC). Annual epidemiological report for 2017. Legionnaires' disease. Stockholm: ECDC; 2019. Available from: https://ecdc.europa. eu/sites/portal/files/documents/AER_for_2017-Legionnairesdisease 0.pdf

4. Beauté JThe European Legionnaires' Disease Surveillance Network. Legionnaires' disease in Europe, 2011 to 2015. Euro Surveill. 2017;22(27). https://doi.org/10.2807/1560-7917. ES.2017.22.27.30566 PMID: 28703097

5. Prussin Al 2nd, Schwake DO, Marr LC. Ten questions concerning the aerosolization and transmission of Legionella in the built environment. Build Environ. 2017;123:68495. https://doi.org/10.1016/j.buildenv.2017.06.024 PMID: 29104349

6. Jernigan DB, Hofmann J, Cetron MS, Genese CA, Nuorti JP, Fields BS, et al. Outbreak of Legionnaires' disease among cruise ship passengers exposed to a contaminated whirlpool spa. Lancet. 1996;347(9000):494-9. https://doi.org/10.1016/ S0140-6736(96)91137-X PMID: 8596266

7. Hicks LA, Rose CE Jr, Fields BS, Drees ML, Engel JP, Jenkins PR, et al. Increased rainfall is associated with increased risk for legionellosis. Epidemiol Infect. 2007;135(5):811-7. https://doi. org/10.1017/S0950268806007552 PMID: 17121693

8. Garcia-Vidal C, Labori M, Viasus D, Simonetti A, GarciaSomoza D, Dorca J, et al. Rainfall is a risk factor for sporadic cases of Legionella pneumophila pneumonia. PLoS One. 2013;8(4):e61036. https://doi.org/10.1371/journal. pone.0061036 PMID: 23613778

9. Chen NT, Chen MJ, Guo CY, Chen KT, Su HJ. Precipitation increases the occurrence of sporadic legionnaires' disease in Taiwan. PLoS One. 2014;9(12):e114337. https://doi. org/10.1371/journal.pone.0114337 PMID: 25474539

10. Rota MC, Caporali MG, Bella A, Scaturro M, Giannitelli S, Ricci ML. La legionellosi in Italia nel 2017. [Legionellosis in Italy in 2017]. Not Ist Super Sanità. 2018;31(9):712. Italian. Available from: https://www.iss.it/ documents/20126/45616/nuovo nov 2019ONLINE 15 .pdf/ ea33e77d-e3a9-1c96-49a8-28dgfdc12506? $t=1581097313618$

11. European Commission. Commission Implementing Decision (EU) $2018 / 945$ of 22 June 2018 on the communicable diseases and related special health issues to be covered by epidemiological surveillance as well as relevant case definitions. Official Journal of the European Union. Luxembourg: Publications Office of the European Union 6.7.2018:L 170/1. Available from: https://eur-lex.europa.eu/ legal-content/EN/TXT/PDF/?uri=CELEX\%3A32018D0945\&from $=$ EN $\% 29$.

12. International Organization for Standardization (ISO). ISO/ TS 12869:2012. Water quality - Detection and quantification of Legionella spp and/or Legionella pneumophila by concentration and genic amplification by quantitative polymerase chain reaction (qPCR). Geneva: ISO; 2019. Available from: https://www.iso.org/standard/52079.html

13. International Organization for Standardization (ISO). ISO 11731. Water Quality. Enumeration of Legionella. Geneva: ISO; 2017. Available from: https://www.iso.org/standard/61782.html

14. Helbig JH, Kurtz JB, Pastoris MC, Pelaz C, Lück PC. Antigenic lipopolysaccharide components of Legionella pneumophila recognized by monoclonal antibodies: possibilities and limitations for division of the species into serogroups. Clin Microbiol. 1997;35(11):2841-5. https://doi.org/10.1128/ JCM.35.11.2841-2845.1997 PMID: 9350744

15. European Working Group for Legionella Infections (EWGLI). The EWGLI SBT database for the typing of Legionella pneumophila. London: Public Health England; 2014. Available from: http:// webarchive.nationalarchives.gov.uk/20190501130700/http:// bioinformatics.phe.org.uk/legionella/legionella_sbt/php/ sbt homepage.php
16. Maclure $M$. The case-crossover design: a method for studying transient effects on the risk of acute events. Am J Epidemiol. 1991;133(2):144-53. https://doi.org/10.1093/oxfordjournals. aje.a115853 PMID: 1985444

17. Levy D, Lumley T, Sheppard L, Kaufman J, Checkoway H. Referent selection in case-crossover analyses of acute health effects of air pollution. Epidemiology. 2001;12(2):186-92. https://doi.org/10.1097/00001648-200103000-00010 PMID: 11246579

18. Breslow N, Day N. Statistical methods in cancer research. Vol I: the analysis of case-control studies. Lyon: International Agency for Research on Cancer (IARC Scientific Publications No. 32); 1980.

19. Lewallen S, Courtright P. Epidemiology in practice: casecontrol studies. Community Eye Health. 1998;11(28):57-8. PMID: 17492047

20. Bellasio R, Bianconi R, Mosca S, Zannetti P. Formulation of the Lagrangian particle model LAPMOD and its evaluation against Kincaid SF 6 and SO 2 datasets. Atmos Environ. 2017;163:8798. https://doi.org/10.1016/j.atmosenv.2017.05.039

21. Scire JS, Robe FR, Fernau ME, Yamartino RJ. A user' s guide for the CALMET meteorological model. Version 5.0. Concord: Earth Tech, Inc; 2000. Available from: http://www.src.com/calpuff/ download/CALMET_UsersGuide.pdf

22. Schoen ME, Ashbolt NJ. An in-premise model for Legionella exposure during showering events. Water Res. 2011;45(18):5826-36. https://doi.org/10.1016/j. watres.2011.08.031 PMID: 21924754

23. Nygård K, Werner-Johansen $\emptyset$, Rønsen S, Caugant DA, Simonsen $\varnothing$, Kanestrøm A, et al. An outbreak of legionnaires disease caused by long-distance spread from an industrial air scrubber in Sarpsborg, Norway. Clin Infect Dis. 2008;46(1):619. https://doi.org/10.1086/524016 PMID: 18171215

24. Llasat MC. An objective classification of rainfall events on the basis of their convective features: application to rainfall intensity in the northeast of spain. Int J Climatol. 2001;21(11):1385-400. https://doi.org/10.1002/joc.692

25. Russo A, Gouveia CM, Soares PMM, Cardoso RM, Mendes MT, Trigo RM. The unprecedented 2014 Legionnaires' disease outbreak in Portugal: atmospheric driving mechanisms. Int J Biometeorol. 2018;62(7):1167-79. https://doi.org/10.1007/ So0484-018-1520-8 PMID: 29572569

26. García-Fulgueiras A, Navarro C, Fenoll D, García J, GonzálezDiego P, Jiménez-Buñuales T, et al. Legionnaires' disease outbreak in Murcia, Spain. Emerg Infect Dis. 2003;9(8):915-21. https://doi.org/10.3201/eido908.030337 PMID: 12967487

27. Castellani Pastoris M, Ciceroni L, Lo Monaco R, Goldoni P, Mentore B, Flego G, et al. Molecular epidemiology of an outbreak of Legionnaires' disease associated with a cooling tower in Genova-Sestri Ponente, Italy. Eur I Clin Microbiol Infect Dis. 1997;16(12):883-92. https://doi.org/10.1007/ BF01700554 PMID: 9495668

28. Rota MC, Pontrelli G, Scaturro M, Bella A, Bellomo AR, Trinito MO, et al. Legionnaires' disease outbreak in Rome, Italy. Epidemiol Infect. 2005;133(5):853-9. https://doi.org/10.1017/ So950268805004115 PMID: 16181505

29. Haupt TE, Heffernan RT, Kazmierczak JJ, Nehls-Lowe H, Rheineck B, Powell C, et al. An outbreak of Legionnaires disease associated with a decorative water wall fountain in a hospital. Infect Control Hosp Epidemiol. 2012;33(2):185-91. https://doi.org/10.1086/663711 PMID: 22227989

30. O'Loughlin RE, Kightlinger L, Werpy MC, Brown E, Stevens V, Hepper C, et al. Restaurant outbreak of Legionnaires' disease associated with a decorative fountain: an environmental and case-control study. BMC Infect Dis. 2007;7(1):93. https://doi. org/10.1186/1471-2334-7-93 PMID: 17688692

31. Beauté J, Sandin S, Uldum SA, Rota MC, Brandsema P, Giesecke J, et al. Short-term effects of atmospheric pressure, temperature, and rainfall on notification rate of communityacquired Legionnaires' disease in four European countries. Epidemiol Infect. 2016;144(16):3483-93. https://doi. org/10.1017/So950268816001874 PMID: 27572105

32. Brandsema PS, Euser SM, Karagiannis I, Den Boer JW, Van Der Hoek W. Summer increase of Legionnaires' disease 2010 in The Netherlands associated with weather conditions and implications for source finding. Epidemiol Infect. 2014;142(11):2360-71. https://doi.org/10.1017/ So950268813003476 PMID: 24576486

33. Heuner K, Swanson M, editors. Legionella: molecular microbiology. Norwich: Caister Academic Press; 2008; 249 p.

34. Agenzia regionale per la protezione dell'ambiente della Lombardia (ARPA Lombardia). Richiesta dati misurati Meteorologia. [Request for measurement data - meteorology.] Milan: ARPA Lombardia. [Accessed: 18 May 2020]. Available from: https://www.arpalombardia.it:443/Pages/Meteorologia/ Richiesta-dati-misurati.aspx 
35. Walker JT. The influence of climate change on waterborne disease and Legionella: a review. Perspect Public Health. 2018;138(5):282-6. https://doi.org/10.1177/1757913918791198 PMID: 30156484

\section{License, supplementary material and copyright}

This is an open-access article distributed under the terms of the Creative Commons Attribution (CC BY 4.0) Licence. You may share and adapt the material, but must give appropriate credit to the source, provide a link to the licence and indicate if changes were made.

Any supplementary material referenced in the article can be found in the online version.

This article is copyright of the authors or their affiliated institutions, 2020. 